R. Achilles, C. Huneke and W. Vogel

Nagoya Math. J.

Vol. 100 (1985), 49-63

\title{
A CRITERION FOR INTERSECTION MULTIPLICITY ONE
}

\section{RÜDIGER ACHILLES, CRAIG HUNEKE AND WOLFGANG VOGEL}

Let $X$ and $Y$ be any pure dimensional subschemes of $\boldsymbol{P}_{K}^{n}$ over an algebraically closed field $K$ and let $I(X)$ and $I(Y)$ be the largest homogeneous ideals in $K\left[x_{0}, \cdots, x_{n}\right]$ defining $X$ and $Y$, respectively. By a pure dimensional subscheme $X$ of $\boldsymbol{P}_{K}^{n}$ we shall always mean a closed pure dimensional subscheme without imbedded components, i.e., all primes belonging to $I(X)$ have the same dimension.

A subvariety $V$ of $\boldsymbol{P}_{K}^{n}$ is a reduced and irreducible closed subscheme of $\boldsymbol{P}_{K}^{n}$; that is, a subvariety corresponds to a prime ideal in $K\left[x_{0}, \cdots, x_{n}\right]$.

The aim of this note is to prove a criterion of multiplicity one for isolated (i.e., irreducible) components $C \subset X \cap Y$. In case of proper components, that is, $\operatorname{dim} C=\operatorname{dim} X+\operatorname{dim} Y-n$, a criterion of multiplicity one is given as a statement on transversality (see, e.g., A. Weil [20], Chapter 6, Theorem 6). Using the approach to the intersection theory of Fulton and MacPherson one obtains a new proof for such a criterion (see W. Fulton [3], Propositions 7.2 and 8.2). However, in [3] we have no observations about a criterion for intersection multiplicity one for improper components.

For improper components of the intersection of subvarieties P. Samuel improves the classical result on transversality (see [12], Chap. II, §5, no. 3). In case of the intersection theory of analytic geometry E. Selder [13] develops such a criterion for proper components of pure dimensional analytic sets. Hence our algebro-geometric proof does yield an algebraization of Selder's consideration. Moreover, our results extend Samuel's and Fulton's observations. Applying our criterion for intersection multiplicity one we see that the problem posed in [17], p. A-4 is not true in general, see our Example 8. Knowing our remark after the proof of Lemma 4 and Fulton's observation in [3], p. 174 (last period) one now expected such examples.

Received April 2, 1984. 
Proving our results we will use the algebraic approach to the intersection theory by [17]. Following this theory we have for every isolated component $C \subset X \cap Y$ an intersection multiplicity, say $j(X, Y ; C) \geq 1$, of $X$ and $Y$ along $C$ given by the length of a well-defined primary ideal (see [17] or consider our Lemma 2 be below). First, we note a result on these new intersection numbers.

Lemma 1. If $\operatorname{dim} C=\operatorname{dim} X+\operatorname{dim} Y-n$, then

$$
j(X, Y ; C)=i(X, Y ; C)
$$

where $i(X, Y ; C)$ is given by Weil's $i$-symbol.

Proof. See [18], Proposition 3.22.

We first introduce some notations. $X \cap Y$ is said to be reduced at a component $C \subset X \cap Y$ if the localization of $I(X)+I(Y)$ at the prime ideal $I(C)$ defining $C$ is given by a prime ideal. Let $A(X ; C)$ and $A(Y ; C)$ be the local rings of $X$ at $C$ and $Y$ at $C$, resp. Then the main result of this note is the following theorem.

Theorem. Let $X$ and $Y$ be pure dimensional subschemes of $\boldsymbol{P}_{K}^{n}$. Let $C$ be any isolated component of $X \cap Y$. Then the following conditions are equivalent:

(i ) $j(X, Y ; C)=1$.

(ii) a) $A(X ; C)$ and $A(Y ; C)$ are regular local rings,

b) $X \cap Y$ is reduced at $C$.

Corollary 1. Assume that the subvarieties $X$ and $Y$ of $\boldsymbol{P}_{K}^{n}$ intersect properly in $C$, then the following conditions are equivalent:

(i) $i(X, Y ; C)=1$.

(ii) $X \cap Y$ is reduced at $C$.

(iii) $X$ and $Y$ are transversal to each other at some point of $C$.

Before embarking on the proof of the theorem and on the construction of examples, we must prove several preliminary results. First we want to recall the definition of the intersection number $j(X, Y ; C)$. Let $X, Y$ be our pure dimensional subschemes of $\boldsymbol{P}_{K}^{n}$ with defining ideals $I(X)$ and $I(Y)$ in $K\left[x_{0}, \cdots, x_{n}\right]=: R_{x}$. We introduce a second copy $K\left[y_{0}, \cdots, y_{n}\right]=$ : $R_{y}$ of $R_{x}$ and denote by $I(Y)^{\prime}$ the ideal in $R_{y}$ corresponding to $I(Y)$. We consider the polynomial ring $R:=K\left[x_{0}, \cdots, x_{n}, y_{0}, \cdots, y_{n}\right]$ and the ideal $\mathfrak{c}=\left(x_{0}-y_{0}, \cdots, x_{n}-y_{n}\right) \cdot R$. Furthermore, we introduce new independent 
variables $u_{i, m}$ over $K ; i, m=0,1, \cdots, n$. Let $\bar{K}$ be the algebraic closure of $K\left(u_{0,0}, \cdots, u_{n, n}\right)$. Put

$$
\bar{R}:=\bar{K}\left[x_{0}, \cdots, x_{n}, y_{0}, \cdots, y_{n}\right] \text {, and } l_{i}:=\sum_{m=0}^{n} u_{i, m}\left(x_{m}-y_{m}\right)
$$

in $\bar{R}$ for $i=0, \cdots, n$.

Let $C$ be an isolated component of $X \cap Y$ with defining prime ideal $I(C)$ of Krull-dimension $j$. We want to construct a well-defined primary ideal belonging to $(I(C)+c) \cdot \bar{R}$ such that $j(X, Y ; C)$ is given by the length of this primary ideal. We set

$\delta:=$ Krull-dimension of $I(X)+I(Y)^{\prime}$ in $\bar{R}$, and

$d:=$ Krull-dimension of $I(X)+I(Y)$ in $R_{x}$.

Take the linear forms $l_{0}, \cdots, l_{\delta-d-1}$ and put

$\left(I(X) \cdot \bar{R}+I(Y)^{\prime} \cdot \bar{R}\right)_{-1}:=I(X) \cdot \bar{R}+I(Y)^{\prime} \cdot \bar{R}$, and

$\left(I(X) \cdot \bar{R}+I(Y)^{\prime} \cdot \bar{R}\right)_{k}:=l_{k} \cdot \bar{R}+U\left(\left(I(X) \cdot \bar{R}+I(Y)^{\prime} \cdot \bar{R}\right)_{k-1}\right)$

for any $k=0, \cdots, \delta-d-1$, where $U(\cdots)$ is the intersection of all highest dimensional primary ideals belonging to the ideal $(\cdots)$. Furthermore, we put

$\mathfrak{a}_{0}:=U\left(\left(I(X) \cdot \bar{R}+I(Y)^{\prime} \cdot \bar{R}\right)_{\delta_{-d-2}}\right)$ and if $j<d$,

$\mathfrak{a}_{s}:=$ intersection of all primary ideals belonging to $U\left(\mathfrak{a}_{s-1}+l_{\delta-d+s-2} \cdot \bar{R}\right)$ such that $c \cdot \bar{R}$ is not contained in their associated primes

for all $s=1, \cdots, d-j$. Following [17] we define

$$
j(X, Y ; C):=\text { length of }\left(\mathfrak{a}_{d-j}+l_{\bar{\delta}-j-1} \cdot \bar{R}\right)_{(I(C)+c) \bar{R}} .
$$

In particular, if $j=d$, then

$$
\left.j(X, Y ; C)=\text { length of }\left((I(X)) \cdot \bar{R}+I(Y)^{\prime} \cdot \bar{R}\right)_{\delta-d-1}\right)_{(I(C)+c) \bar{R}} .
$$

We need a main lemma of [18], (3.19). Let $C$ be any isolated component of $X \cap Y$ in $\boldsymbol{P}_{K}^{n}$ of Krull-dimension $j$. We set $\bar{A}:=(\bar{R} / I(X)+$ $\left.I(Y)^{\prime}\right)_{I(C)+c}$; that is, $\bar{A}$ is the local ring of the "join-variety" of $X$ and $Y$ in $\boldsymbol{P}_{\bar{K}}^{2 n+1}$ at the corresponding subvariety defined by $I(C)+c$. Hence $\delta-$ $j=$ Krull-dimension of $\bar{A}$. We put $l(X, Y ; C):=$ length of $\left(\boldsymbol{R}_{x} / I(X)+\right.$ $I(Y))_{I(C)}$.

Lemma 2. $j(X, Y ; C)=l(X, Y ; C)+k_{2}-k_{1}$ where

$k_{2}=$ length of $\mathrm{c} \cdot \bar{A} /\left(l_{0}, \ldots, l_{\hat{\delta}-j-1}\right) \cdot \bar{A}$ over $\bar{A}$, and

$k_{1}=$ length of $\left(l_{0}, \cdots, l_{\delta-j-2}\right):_{\bar{A}} l_{\hat{\delta}-j-1} /\left(l_{0}, \cdots, l_{\delta-j-2}\right) \cdot \bar{A}$ over $\bar{A}$.

Proof. See [18], Corollary (3.19).

Recall that if $B=S / I$ is a local ring where $S$ is a regular local ring 
with maximal ideal $\mathfrak{n}$ and $I$ an ideal of $S$,

$$
d(B)=\operatorname{dim}_{S / \mathfrak{n}} I / \mathfrak{n} I-\text { height } I
$$

is called the complete intersection defect of $B$. It is $d(B) \geq 0$, and equality holds if and only if $B$ is a complete intersection; that is, $I$ is generated by a regular sequence (cf. [7] and [1] for the definition and basic properties of the complete intersection defect). In a Cohen-Macaulay local ring $A$ with maximal ideal $\mathfrak{m}$ the number of irreducible components of an ideal generated by a system of parameters is an invariant $r(A)$ of the ring and is called the type of $A$; it is $r(A)=\operatorname{dim}_{A / \mathfrak{m}} \operatorname{Ext}_{A}^{d}(A / \mathfrak{m}, A)$ where $d=$ $\operatorname{dim} A$ (see, e.g., [6], 1.20 and 1.34). $A$ is Gorenstein if and only if it is of type one. Now we are ready to state our second key lemma.

LEMMA 3. Let $\mathscr{P}$ be one of the following properties of a noetherian local ring: (a) regularity, (b) complete intersection, (c) Gorenstein, (d) Cohen-Macaulay.

Then the following conditions are equivalent:

(i) $\bar{A}$ has $\mathscr{P}$.

(ii) $A(X ; C)$ and $A(Y ; C)$ have $\mathscr{P}$.

Furthermore, for the complete intersection defect it holds

$$
d(\bar{A})=d(A(X ; C))+d(A(Y ; C)),
$$

and if $\bar{A}$ is Cohen-Macaulay, for the type one has

$$
r(\bar{A})=r(A(X ; C)) \cdot r(A(Y ; C)) .
$$

When proving Lemma 3 we can forget about the field extension of $K$ to $\bar{K}$ as the next lemma shows.

Lemma 4. If $A:=\left(R / I(X)+I(Y)^{\prime}\right)_{I(C)+\mathrm{c}}$ and $e_{0}(\mathfrak{q}, A)$ denotes the multiplicity of an m-primary ideal $\mathfrak{q}$ of the local ring $A$ with maximal ideal $m$ (see, e.g., [21], Vol. II, Chap. VIII, §10), then $\mathfrak{m} \cdot \bar{A}$ is the maximal ideal of $\bar{A}, \mathfrak{q} \cdot \bar{A}$ is an $\mathfrak{m} \cdot \bar{A}$-primary ideal and

$$
e_{0}(\mathfrak{q}, A)=e_{0}(\mathfrak{q} \cdot \bar{A}, \bar{A}) .
$$

Furthermore, if $\mathscr{P}$ is a property as in Lemma $3, A$ has $\mathscr{P}$ if and only if $\bar{A}$ has $\mathscr{P}, d(A)=d(\bar{A})$ and, in case $A$ is Cohen-Macaulay, $r(A)=r(\bar{A})$.

Proof. The extension to $\bar{R}$ of a prime ideal of $R$ is prime (cf. [21], Vol. II, Chap. VII, § 11, Cor. on p. 226 and Thm. 38). Therefore it is obvious that $\mathfrak{m} \cdot \bar{A}$ is the maximal ideal of $\bar{A}$, and $\mathfrak{q} \cdot \bar{A}$ is $\mathfrak{m} \cdot \bar{A}$-primary. Using $\mathfrak{q}$ 
$=A \cap \mathfrak{q} \cdot \bar{A}$ (cf.[12], Chap. II, $\S 1$, no 3 ; for $\mathfrak{q}$ one concludes in the same way as it has been done there for $\mathrm{m}^{n}$ ) one can see that a composition series of $q$ extends to one of $q \cdot \bar{A}$. Thus the Hilbert-Samuel functions of $q$ and $\mathfrak{q} \cdot \bar{A}$ coincide, and in particular we have $e_{0}(\mathfrak{q}, A)=e_{0}(\mathfrak{q} \cdot \bar{A}, \bar{A})$.

Since $A$ and $\bar{A}$ are unmixed (see, e.g., [10], (34.10)), they are regular if and only if their multiplicity is one ([10], (40.6)). Hence the equality $e_{0}(\mathfrak{m}, A)=e_{0}(\mathfrak{m} \cdot \bar{A}, \bar{A})$ implies that $A$ is regular if and only if $\bar{A}$ is so. Concerning the proof of the statement about the other properties $\mathscr{P}$ and the invariants $d$ and $r$ see [5], 6.7.1 for the Cohen-Macaulay property and [1], (3.14) for the complete intersection property and for $d$. Using the behaviour of the type under local flat extensions (see [6], 1.24), in the same way the proof for the type, and in particular for the Gorenstein property, is obtained.

Remark. If $C$ is an isolated component of $X \cap Y$, then $j(X, Y ; C)=$ $e_{0}(\mathfrak{c} \cdot \bar{A}, \bar{A})$ (see [11], (2.3) and [0], Prop. 1). Hence $j(X, Y ; C)=e_{0}(\mathfrak{c} \cdot A, A)$ by Lemma 4 ; that is, $j(X, Y ; C)$ coincides with Samuel's intersection multiplicity of (irreducible and reduced) $X$ and $Y$ along $C$ defined in [12], Chap. II, $\S 5$, no. 1 .

Proof ${ }^{1)}$ of Lemma 3. We abbreviate $I(X)=\mathfrak{a}, I(Y)=\mathfrak{b}, I(C)=\mathfrak{p}$, $K\left[x_{0}, \cdots, x_{n}\right]=K[x], K\left[y_{0}, \cdots, y_{n}\right]=K[y]$. Clearly the obvious homomorphism of rings $K \rightarrow K[x] / \mathfrak{a}$ is flat. In the same way $K[y] / \mathfrak{b}^{\prime}$ is a flat $K$-module. Hence by change of base ([9], (3.C)) we obtain that $K[x] / \mathfrak{a} \otimes_{K}$ $K[y] / \mathfrak{b}^{\prime} \cong K[x, y] / \mathfrak{a}+\mathfrak{b}^{\prime}$ is a flat $K[x] / \mathfrak{a}$-module. From this one derives the following local flat homomorphism

$$
f: A(X ; C)=(K[x] / \mathfrak{a})_{\mathfrak{p}} \longrightarrow\left(K[x, y] / \mathfrak{a}+\mathfrak{b}^{\prime}\right)_{\mathfrak{p}+\mathfrak{c}}=A
$$

because the prime ideal $\mathfrak{p}+\mathfrak{c}$ of $K[x, y] / \mathfrak{a}+\mathfrak{b}^{\prime}$ contracts to $\mathfrak{p}$ in $K[x] / \mathfrak{a}$ (see [9], (3.J)). Let $F$ denote the fibre over the maximal ideal in $A(X ; C)$ of the local flat homomorphism $f$, i.e., $F=\left(K[x, y] / \mathfrak{p}+\mathfrak{b}^{\prime}\right)_{p+c}$. It is known that $A(X ; C)$ (and similarily $A(Y ; C)$ ) has $\mathscr{P}$ if $A$ has $\mathscr{P}$, and if $A(X ; C)$ and $F$ have $\mathscr{P}$, then so does $A$ (see [9], (21.D) for (a); [1], (1.9.2) for (b); [6], 1.24 for (c); and [9], (21. C) for (d)). For the complete intersection defect it holds

$$
d(A)=d(A(X ; C))+d(F)
$$

1) Originally the first author proved Lemma 3 (only for the Cohen-Macaulay property) by using complete tensor products. He thanks Christel Rotthaus for the idea of the present proof, which uses local flat extensions. 
([1], (3.6)), and if $A$ is Cohen-Macaulay, for the type one has

$$
r(A)=r(A(X ; C)) \cdot r(F)
$$

([6], 1.24). In view of Lemma 4 it remains to be shown that $F$ has $\mathscr{P}$ if and only if $A(Y ; C)$ does so and that $d(F)=d(A(Y ; C))$ and, if $F$ is CohenMacaulay, $r(F)=r(A(Y ; C))$. We regard a second local flat homomorphism

$$
g: A(Y ; C)=\left(K[y] / \mathfrak{b}^{\prime}\right)_{\mathfrak{p}^{\prime}} \longrightarrow\left(K[x, y] / \mathfrak{p}+\mathfrak{b}^{\prime}\right)_{\mathfrak{p}+\mathfrak{c}}=F
$$

which is obtained in the same way as $f$. The fibre of $g$ over the maximal ideal of $A(Y ; C)$ is $\left(K[x, y] / \mathfrak{p}+\mathfrak{p}^{\prime}\right)_{\mathfrak{p}+\mathfrak{c}}=: B$. We shall prove the following claim.

ClaIm: If $K$ is a perfect field and $\mathfrak{p} \subseteq K[x]$ is a prime ideal, then $B=\left(K[x, y] / \mathfrak{p}+\mathfrak{p}^{\prime}\right)_{\mathfrak{p}+\mathfrak{c}}$ is a regular local ring.

(Note, that the claim is not true in general if $K$ is not perfect.) Having this and using the behaviour of $\mathscr{P}, d$, and $r$ under a local flat homomorphism again, it follows that $A(Y ; C)$ has $\mathscr{P}$ if and only if $F$ does so and that $A(Y ; C)$ and $F$ have the same complete intersection defect and, if they are Cohen-Macaulay, the same type.

So let us prove our claim. Let $j$ denote the Krull-dimension of $K[x] / \mathfrak{p}$. Then there are $j$ independent indeterminates with respect to $\mathfrak{p}$, say $x_{0}, \cdots$, $x_{j-1}$, while $x_{0}, \cdots, x_{j-1}, x_{i}$ are dependent for every $i=j, \cdots, n$; that is, there is a polynomial in $\mathfrak{p}$ depending only on $x_{0}, \cdots, x_{j-1}, x_{i}$ (cf. [4], 131.7). We will show that the $j$ elements $x_{0}-y_{0}, \cdots, x_{j-1}-y_{j-1}$ (regarded as elements of $B$ ) generate the maximal ideal of the $j$-dimensional local ring $B$. Since $K$ is perfect, by [19], $\S 155$ (or [21], Vol. I, chap. II, $\S 13$, Theorem 31) applied to the quotient field of $K[x] / p$, for every $i=j, \cdots, n$ there is a non-zero polynomial $f_{i}\left(x_{0}, \cdots, x_{j-1}, x_{i}\right) \in \mathfrak{p}$ depending only on $x_{0}, \cdots, x_{j-1}$, $x_{i}$, which is irreducible and separable as a polynomial in $x_{i}$. Clearly $f_{i}\left(x_{0}, \cdots, x_{j-1}, x_{i}\right)$ is a polynomial in $\mathfrak{p}$ of smallest possible positive degree in $x_{i}$. We have

$$
\text { (*) } \quad \begin{aligned}
f_{i}\left(x_{0}, \cdots, x_{j-1}, x_{i}\right)-f_{i}\left(x_{0}, \cdots, x_{j-1}, y_{i}\right) \\
\quad=\left(x_{i}-y_{i}\right) \cdot g_{i}\left(x_{0}, \cdots, x_{\jmath-1}, x_{i}, y_{i}\right) \in\left(x_{0}-y_{0}, \cdots, x_{j-1}-y_{j-1}, \mathfrak{p}, \mathfrak{p}^{\prime}\right) B .
\end{aligned}
$$

Note that since $f_{i}$ is separable, it contains at least one monom whose exponent $n$ of $x_{i}$ is not divisible by the characteristic of $K$. Hence

$$
\left(x_{i}^{n}-y_{i}^{n}\right) /\left(x_{i}-y_{i}\right)=x_{i}^{n-1}+x_{i}^{n-2} y_{i}+\cdots+y_{i}^{n-1}
$$


does not contain $x_{\imath}-y_{i}$ as a factor, whence $g_{i}\left(x_{0}, \cdots, x_{j-1}, x_{i}, x_{i}\right) \neq 0$. Since the degree in $x_{i}$ of the latter polynomial is strictly smaller than that of $f_{i}$, we have $g_{i}\left(x_{0}, \cdots, x_{j-1}, x_{i}, x_{i}\right) \notin p$ and therefore $g_{i}\left(x_{0}, \cdots, x_{j-1}\right.$, $\left.x_{i}, y_{i}\right) \notin \mathfrak{p}+$ c. So $g_{i}\left(x_{0}, \cdots, x_{j-1}, x_{i}, y_{i}\right)$ is a unit in $B$, and from $(*)$ we get that

$$
x_{i}-y_{i} \in\left(x_{0}-y_{0}, \cdots, x_{j-1}-y_{j-1}, \mathfrak{p}, \mathfrak{p}^{\prime}\right) B \quad \text { for all } i=j, \cdots, n .
$$

This establishes our claim and finishes the proof of Lemma 3.

Proof of the theorem. (i) $\Rightarrow$ (ii): It follows from [11], (2.3) that $j(X, Y ; C)$ $=e_{0}(\mathfrak{c} \cdot \bar{A}, \bar{A})$ where $e_{0}(\bar{q}, \bar{A})$ denotes the multiplicity of an $\overline{\mathfrak{m}}$-primary ideal $\overline{\mathfrak{q}}$ in the local ring $\bar{A}$ with maximal ideal $\overline{\mathfrak{m}}$. Hence we obtain

$$
1=j(X, Y ; C)=e_{0}(\mathfrak{c} \cdot \bar{A}, \bar{A}) \geq e_{0}(\overline{\mathfrak{m}}, \bar{A}) \geq 1 ;
$$

that is, $e_{0}(\overline{\mathfrak{m}}, \bar{A})=1$. Since $X$ and $Y$ are pure dimensional subschemes, it follows from [10], (34.10) that $\bar{A}$ is unmixed (note that $\bar{A}$ is a homomorphic image of a regular local ring). We therefore get from [10], (40.6) that $\bar{A}$ is regular. Hence by (**) we have $c \cdot \bar{A}=\overline{\mathfrak{n}}$; that is, $X \cap Y$ is reduced at $C$. Lemma 3 , (a) does yield the condition (ii), (a) of the theorem, and the implication

(i) $\Rightarrow$ (ii) is proved.

(ii) $\Rightarrow$ (i): Since $X \cap Y$ is reduced at $C$ we have $c \cdot \bar{A}=\overline{\mathfrak{m}}$. The theorem (2.3) or [11] does provide that $j(X, Y ; C)=e_{0}(c \cdot \bar{A}, \bar{A})$. Hence we obtain

$$
j(X, Y ; C)=e_{0}(\mathfrak{c} \cdot \bar{A}, \bar{A})=e_{0}(\overline{\mathfrak{m}}, \bar{A})=1
$$

since $\bar{A}$ is regular by applying Lemma 3 , (a). This proves our theorem.

Before proving our Corollary 1 we want to improve Serre's results on the relation between multiplicities and lengths by using our approach. The statement is the following (see also [18], Corollary (3.20)):

Proposition. Let $X, Y$ be pure dimensional subschemes of $\boldsymbol{P}_{k}^{n}$. Let $C$ be any isolated component of $X \cap Y$. Then we have:

(i) Assume that $A(X ; C)$ and $A(Y ; C)$ are Cohen-Macaulay. Then

$$
j(X, Y ; C) \geq l(X, Y ; C)
$$

and equality holds if and only if $\mathfrak{c} \cdot \bar{A} \subseteq\left(l_{0}, \cdots, l_{\delta-j-1}\right) \cdot \bar{A}$.

(ii) Suppose that $c \cdot \bar{A} \subseteq\left(l_{0}, \cdots, l_{\delta-j-1}\right) \cdot \bar{A}$. Then

$$
j(X, Y ; C) \leq l(X, Y ; C)
$$


and equality holds if and only if $A(X ; C)$ and $A(Y ; C)$ are Cohen-Macaulay.

Proof. The assertions (i) and (ii) result immediately from Lemma 2 and Lemma $3,(d)$.

The Proposition does yield new proofs for Serre's and Gröbner's results on lengths and multiplicities, see [14], Chapter V, [4], 144.12 and [2].

Corollary 2. The notations being the same as in the Proposition.

(i) Suppose that $\operatorname{dim} C=\operatorname{dim} X+\operatorname{dim} Y-n$, then

$$
i(X, Y ; C) \leq l(X, Y ; C)
$$

and equality holds if and only if $A(X ; C)$ and $A(Y ; C)$ are Cohen-Macaulay. Assume that $\operatorname{dim} X \cap Y=\operatorname{dim} X+\operatorname{dim} Y-n$, then

$$
\operatorname{deg}(X) \cdot \operatorname{deg}(Y) \leqq \operatorname{deg}(X \cap Y) .
$$

(ii) Assume that $A(X ; C)$ and $A(Y ; C)$ are Cohen-Macaulay, then

$$
j(X, Y ; C) \geq l(X, Y ; C) \text {. }
$$

Suppose that for every component $C \subset X \cap Y$ with $\operatorname{dim} C=\operatorname{dim} X \cap Y$ the local rings $A(X ; C)$ and $A(Y ; C)$ are Cohen-Macaulay, then

$$
\operatorname{deg}(X) \cdot \operatorname{deg}(Y) \geq \operatorname{deg}(X \cap Y) \text {. }
$$

(iii) Assume that $\operatorname{dim} X \cap Y=\operatorname{dim} X+\operatorname{dim} Y-n$. Then $A(X ; C)$ and $A(Y ; C)$ are Cohen-Macaulay for every isolated component $C \subset X \cap Y$ if and only if $\operatorname{deg}(X) \cdot \operatorname{deg}(Y)=\operatorname{deg}(X \cap Y)$.

Proof. We note the following two facts: If $\operatorname{dim} C=\operatorname{dim} X+\operatorname{dim} Y$ $-n$ then $\delta-j-1=n$; that is, $\left(l_{0}, \cdots, l_{\delta-j-1}\right) \cdot \bar{A}=c \cdot \bar{A}$. Furthermore, $\operatorname{deg}(X \cap Y)=\sum_{c} l(X, Y ; C) \cdot \operatorname{deg}(C)$, where $C$ runs through all irreducible components of $X \cap Y$ such that $\operatorname{dim} C=\operatorname{dim} X \cap Y$. Having these facts, the Corollary follows immediately from the proposition and Lemma 1.

Corollary 3. Assume that the local rings $A(X ; C)$ and $A(Y ; C)$ are Cohen-Macaulay for all components $C \subset X \cap Y$ with $\operatorname{dim} C=\operatorname{dim} X \cap Y$. Then the following conditions are equivalent:

(i) $\operatorname{deg}(X) \cdot \operatorname{deg}(Y)=\operatorname{deg}(X \cap Y)$.

(ii) The collection $\left\{C_{i}\right\}$ of the theorem of [17] is equal to $\{C \mid C \subset X$ $\cap Y$ and $\operatorname{dim} C=\operatorname{dim} X \cap Y\}$, and $j(X, Y ; C)=l(X, Y ; C)$ for all $C \subset X \cap Y$ with $\operatorname{dim} C=\operatorname{dim} X \cap Y$.

Proof. The theorem of [17] and our Lemma 2 do yield 


$$
\begin{aligned}
\operatorname{deg}(X) \cdot \operatorname{deg}(Y)= & \sum_{C_{i} \in\left\{C_{i}\right\}} j(X, Y ; C) \cdot \operatorname{deg}\left(C_{i}\right) \geq \sum_{\substack{C \in X \cap Y \\
\operatorname{dim} C=\operatorname{dim} X \cap Y}} j(X, Y ; C) \cdot \operatorname{deg}(C) \\
& \geq \sum l(X, Y ; C) \cdot \operatorname{deg}(C)=\operatorname{deg}(X \cap Y)
\end{aligned}
$$

From this our corollary immediately follows.

This corollary yields the following interesting result.

Corollary 4. Let the situation be as described in Corollary 3. Then every isolated component of $X \cap Y$ has the same dimension as $X \cap Y$ if $\operatorname{deg}(X) \cdot \operatorname{deg}(Y)=\operatorname{deg}(X \cap Y)$.

One special case of Corollary 4 is the following remark:

Remark. Assume that $X$ and $Y$ are locally Cohen-Macaulay (for example, $X$ and $Y$ non-singular). Suppose that $\operatorname{deg}(X \cap Y)=\operatorname{deg}(X)$. $\operatorname{deg}(Y)$, then $X \cap Y$ has no isolated components of dimension $<\operatorname{dim} X \cap Y$.

Proof of Corollary 1. Lemma 1 does yield that $j(X, Y ; C)=i(X, Y ; C)$. Hence we can apply our theorem and Corollary 2, (i).

(i ) $\Longleftrightarrow$ (iii) is well-known; see, for example, [20], Chapter VI, Theorem 6.

(i) $\Longrightarrow$ (ii) results immediately from the theorem.

(ii) $\Longrightarrow$ (i ): Since $X \cap Y$ is reduced at $C$ we get that $l(X, Y ; C)=1$. Hence we obtain $1 \leq i(X, Y ; C) \leq l(X, Y ; C)=1$ by applying Corollary 2 , (i). This proves Corollary 1 .

Now we will show by examples that the above theorem does not remain true when the condition (a) or (b) of (ii) is dropped. This is clear for condition (b) by studying Corollary 1. Before considering our examples we collect some helpful results that we need. First, the following result (see also [12], Chap. II, $\S 6$, no. 2, a)) can be derived immediately from the theorem:

Corollary 5. Let $X$ be a pure dimensional subscheme of $\boldsymbol{P}^{n}$. Assume that $Y$ is a subvariety of $X$. Then $j(X, Y ; Y)=1$ if and only if $A(X ; Y)$ is regular.

The following example shows that the intersection multiplicity depends on the characteristic of the ground field.

Example 1. Let $X=Y$ be a circle in $\boldsymbol{P}_{K}^{2}$. If char $K \neq 2$ then $X$ is irreducible. It follows from Corollary 5 that $j(X, X ; X)=1$. If char $K=2$ 
then $X$ degenerates into a double line. The theorem shows that $j(X, X ; X)$ $\geq 2$.

Analyzing our proposition we see that the crucial point is to study the condition $c \cdot \bar{A} \subseteq\left(l_{0}, \cdots, l_{\hat{o}-j-1}\right) \cdot \bar{A}$. Therefore we want to investigate this problem in the following two lemmas.

Lemma 5. The notations being the same as in the proposition. Let $X \cap Y$ be reduced at $C$. Then $k_{2}=0$ if and only if $A(X ; C)$ and $A(Y ; C)$ are regular local rings.

Proof. By Lemma 3, (a), it is enough to show that $k_{2}=0$ if and only if $\bar{A}$ is a regular local ring. One has immediately from the definition of $k_{2}$ (see Lemma 2) that $k_{2}=0$ is equivalent to $\left(l_{0}, \cdots, l_{\delta-j-1}\right) \cdot \bar{A}=c \cdot \bar{A}$, where $l_{0}, \ldots, l_{\hat{0}-j-1}$ is a system of parameters for $\bar{A}$. Now by assumption $X \cap Y$ is reduced at $C$, that is $c \cdot \bar{A}=\overline{\mathfrak{m}}$. Hence $k_{2}$ vanishes if and only if $\left(l_{0}, \cdots\right.$, $\left.l_{\delta-j-1}\right) \cdot \bar{A}=\overline{\mathrm{m}}$. But the latter equality is equivalent to the regularity of $\bar{A}$. Sufficiency is clear. Conversely, if $\bar{A}$ is regular, then $\overline{\mathfrak{m}}=\left(l_{0}, \cdots, l_{n}\right)$. $\bar{A}=\mathfrak{c} \cdot \bar{A}$ can be generated by any $\delta-j$ elements of the $l$ 's. This proves the lemma.

Lemma 6. The notations being the same as in the proposition. Assume that $A(X ; C)$ and $A(Y ; C)$ are Cohen-Macaulay and $k_{2}=0$. Then for the complete intersection defect of $X \cap Y$ at $C$ it holds

$$
d\left(\left(R_{x} / I(X)+I(Y)\right)_{I(C)}\right)=d(A(X ; C))+d(A(Y ; C)) .
$$

In particular, if $A(X ; C)$ and $A(Y ; C)$ are complete intersections and $k_{2}=0$, then $X \cap Y$ is a complete intersection at $C$; that is, the primary ideal of $I(X)+I(Y)$ at the localization of the prime ideal $I(C)$ is generated by a regular sequence.

Proof. Because of $d(\bar{A})=d(A(X ; C))+d(A(Y ; C))$ (Lemma 3) it suffices to show that $d(\bar{A})=d\left(\left(R_{x} / I(X)+I(Y)\right)_{I(C)}\right)$. By Lemma 3, $(d)$, our assumptions ensure that $\bar{A}$ is Cohen-Macaulay and, since $k_{2}=0$, that $\left(l_{0}, \cdots, l_{\hat{\delta}-j-1}\right) \cdot \bar{A}=c \cdot \bar{A}$, where $l_{0}, \cdots, l_{\hat{0}-j-1}$ is a system of parameters for $\bar{A}$. By [1], (3.10), this implies

$$
d(\bar{A})=d\left(\bar{A} /\left(l_{0}, \cdots, l_{\delta-j-1}\right) \cdot \bar{A}\right)=d(\bar{A} / \mathfrak{c} \cdot \bar{A}) .
$$

But $d(\bar{A} / \mathfrak{c} \cdot \bar{A})=d(A / \mathfrak{c} \cdot A)$ (see [1], (3.14)) and $A / \mathfrak{c} \cdot A \cong\left(R_{x} / I(X)+I(Y)\right)_{I(C)}$, which completes the proof. 
Lemma 6 and Lemma 2 yield the following corollary:

Corollary 7. Let $X, Y$ be pure dimensional subschemes of $\boldsymbol{P}_{K}^{n}$ and let $C$ be an isolated component of $X \cap Y$. Assume that the local rings $A(X ; C)$ and $A(Y ; C)$ are regular. Then the following conditions are equivalent:

(i ) $j(X, Y ; C)=l(X, Y ; C)$.

(ii) $X \cap Y$ is a complete intersection at $C$.

(iii) $k_{2}=0$.

Proof. We have only to show that the condition (ii) implies (iii). Since $\left(R_{x} / I(X)+I(Y)\right)_{I(C)} \cong A / \mathfrak{c} \cdot A$, it follows from (ii) that $\bar{A} / c \cdot \bar{A}$ is a complete intersection, too. Using Lemma 3 we get that $\bar{A}$ is regular. Therefore $c \cdot \bar{A}$ can be generated by a regular sequence. Hence every minimal system of generators for $c \cdot \bar{A}$ is a regular sequence. Since $c \cdot \bar{A}$ $=\left(l_{0}, \cdots, l_{n}\right) \cdot \bar{A}, \delta-j$ (where $j=$ Krull-dimension of $C$ ) linear forms, say $l_{i 0}, \cdots, l_{i_{\delta-j-1}}$, of $l_{0}, \cdots, l_{n}$ generate the ideal $c \cdot \bar{A}$. The definition of the intersection multiplicity $j(X, Y ; C)$ shows (see the algorithm of [17]) that by renumbering of the linear forms $l_{0}, \cdots, l_{n}$ we get

$$
k_{2}=\text { length of } \mathfrak{c} \cdot \bar{A} /\left(l_{i_{0}}, \cdots, l_{i_{\delta-\jmath}-1}\right) \cdot \bar{A} .
$$

This provides the condition (iii).

Example 2. In $P_{K}^{3}$ we consider the curve $X$ and surface $Y$ defined by the following ideals:

$I(X):=\left(x_{0} x_{2}-x_{1}^{2}, x_{1} x_{3}-x_{2}^{2}\right)=I(C) \cap\left(x_{1}, x_{2}\right)$, where $I(C)$ is the defining ideal of the twisted cubic curve in $P_{K}^{3}$, and $I(Y):=\left(x_{0} x_{3}-x_{1} x_{2}\right)$. Then $X \cap Y$ is given by $I(X)+I(Y)=I(C)$; that is, $X \cap Y$ is reduced at $C$ but it is not a complete intersection at $C$. Of course, $A(X ; C)$ and $A(Y ; C)$ are complete intersections. Hence we get from Lemma 6 that $k_{2} \neq 0$. Furthermore, we obtain $k_{1}=0$. Therefore Lemma 2 does yield that $j(X, Y$; C) $\geq 2$.

This example shows that we cannot drop the condition (ii), a) of the theorem. Analyzing the proof of our proposition we see that we have studied the condition $k_{1}=0$ or $k_{2}=0$. But Lemma 2 shows that the key is to study the difference between $k_{2}$ and $k_{1}$. Therefore we want to give three examples with $k_{1}=k_{2} \neq 0, k_{1}>k_{2} \neq 0$ and $k_{2}>k_{1} \neq 0$ for improper components $C \subset X \cap Y$.

Example 3. In $P_{K}^{4}$ we take subschemes $X$ and $Y$ defined by the folowing ideals: 
$I(X)=\left(x_{0}, x_{1}\right) \cap\left(x_{2}, x_{3}\right)$ and $I(Y)=\left(x_{0}, x_{2}, x_{1}+x_{3}\right)$. Then $X \cap Y$ is given by $I(X)+I(Y)=\left(x_{0}, x_{2}, x_{1} x_{3}, x_{1}+x_{3}\right)$; that is, $X \cap Y$ has only one isolated component $C$ defined by $I(C)=\left(x_{0}, x_{1}, x_{2}, x_{3}\right)$. Using the algorithm from [17] it is not too hard to see that $j(X, Y ; C)=2$. By Lemma 2, we have $2=j(X, Y ; C)=l(X, Y ; C)+k_{2}-k_{1}$, and since $l(X, Y ; C)=2$ it follows that $k_{2}=k_{1}$. Since $A(X ; C)$ is not Cohen-Macaulay, we obtain from Lemma 2 (definition of $k_{1}$ ) and Lemma 3 , (d) that $k_{1} \neq 0$.

Example 4. In $P_{K}^{4}$ we consider subschemes $X$ and $Y$ defined by the following ideals:

$$
I(X)=\left(x_{0}, x_{1}\right) \cap\left(x_{2}, x_{3}\right) \text { and } I(Y)=\left(x_{0}+x_{2}, x_{1}+x_{3}, x_{0}+x_{3}\right), \text { Then } X \cap Y
$$
has only one isolated component, say $C$, given by the ideal $I(C)=\left(x_{0}, x_{1}\right.$, $\left.x_{2}, x_{3}\right)$. It is easy to see that $l(X, Y ; C)=5$. Since $A(X ; C)$ is not CohenMacaulay we get $k_{1} \neq 0$, and $j(X, Y ; C) \geq 2$ by the theorem. The main theorem of [17] does yield $2=\operatorname{deg}(X) \cdot \operatorname{deg}(Y) \geq j(X, Y ; C) \cdot \operatorname{deg}(C)$. Hence we obtain $j(X, Y ; C)=2$. Lemma 2 does provide $2=j(X, Y ; C)=l(X, Y ; C)$ $+k_{2}-k_{1}=5+k_{2}-k_{1}$; that is, $k_{1}>k_{2}$. It is not too hard to see that $k_{2} \neq 0$ by studying the corresponding module defining $k_{2}$.

Example 5. In $P_{K}^{4}$ we consider the cone $X$ over the rational quartic curve given parametrically by $\left\{s^{4}, s^{3} t, s t^{3}, t^{4}\right\}$ in $P^{3}$. Let $Y$ be the vertex of $X$ given by the ideal $I(Y)=\left(x_{0}, x_{1}, x_{2}, x_{3}\right)$; that is, $Y \subset X$. Then $X \cap Y$ is reduced at $C=Y$. Hence we have $l(X, Y ; C)=1$. Since $A(X ; Y)$ is not Cohen-Macaulay, we get that $k_{1} \neq 0$ and $j(X, Y ; Y) \geq 2$ by applying Corollary 5. Lemma 2 does yield $2 \leq j(X, Y ; Y)=l(X, Y ; Y)+k_{2}-k_{1}=1+k_{2}$ $-k_{1}$. Therefore we obtain $k_{2}>k_{1} \neq 0$.

We think that this note and the papers [17], [11] and [18] do yield a good understanding for the importance of the isolated components to the intersection theory. Therefore the following very interesting question remains open (see also [18]): How do imbedded components contribute to the intersection multiplicities?

We believe that the history of this problem is rather interesting. In 1908, Severi [15] discovered a method for an intersection theory including the case of improper components. In 1947, Severi [16] "showed" by using this method that one obtains for every isolated component $C \subset X \cap Y$ a well-defined intersection multiplicity, say $s(X, Y ; C) \geq 0$, such that

$$
\operatorname{deg}(X) \cdot \operatorname{deg}(Y)=\sum_{C} s(X, Y ; C) \cdot \operatorname{deg}(C),
$$


where $C$ runs through all isolated components of $X \cap Y$. Counterexamples were given by Fulton [3] and by Lazarsfeld [8], too. Therefore we want to recall the conjecture of [18], Chapter III, $C$ :

Conjecture. Let $X$ and $Y$ be pure dimensional subschemes of $\boldsymbol{P}_{K}^{n}$. Then $\operatorname{deg}(X) \cdot \operatorname{deg}(Y) \geq$ number of all prime ideals $\mathfrak{p}$ belonging to $I(X)$ $+I(Y)$ such that $\operatorname{dim} \mathfrak{p} \geq \operatorname{dim} X+\operatorname{dim} Y-n$.

Analyzing the algebraic approach of [17] and this conjecture, one might be tempted to ask the following question: Let $C \subset X \cap Y$ be an irreducible subvariety corresponding to an imbedded prime ideal belonging to $I(X)+I(Y)$. If $\operatorname{dim} C \geq \operatorname{dim} X+\operatorname{dim} Y-n$ and if $\operatorname{deg}(X) \cdot \operatorname{deg}(Y)>$ $\sum_{C} j(X, Y ; C) \cdot \operatorname{deg}(C)$, where $C$ runs through all isolated components of $X \cap Y$, then we need the component $C$ for our decomposition of Bezout's number $\operatorname{deg}(X) \cdot \operatorname{deg}(Y)$. However, this is not so, as we will show by the following example.

Example 6: Let $X$ and $Y$ be the surfaces of $P_{K}^{4}$ with the following ideals:

$$
\begin{aligned}
& I(X)=\left(x_{0}, x_{1}\right) \cap\left(x_{0}, x_{2}\right) \cap\left(x_{2}, x_{3}\right), \text { and } \\
& I(Y)=\left(x_{1}, x_{4}\right) \cap\left(x_{0}^{2}, x_{0}+x_{2}\right) .
\end{aligned}
$$

Then we have the following primary decomposition:

$$
I(X)+I(Y)=\left(x_{0}, x_{2}\right) \cap\left(x_{0}, x_{1}, x_{4}\right) \cap\left(x_{1}, x_{2}, x_{3}, x_{4}\right) \cap\left(x_{0} x_{2}, x_{0}+x_{2}, x_{1}, x_{3}\right) .
$$

Applying the algebraic approach of [17] it is not too difficult to show that our decomposition of the Bezout number $\operatorname{deg}(X) \cdot \operatorname{deg}(Y)$ is given by

$$
\operatorname{deg}(X) \cdot \operatorname{deg}(Y)=\sum_{i=1}^{6} j(X, Y ; C) \cdot \operatorname{deg}\left(C_{i}\right),
$$

where the components $C_{i} \subset X \cap Y$ and the intersection multiplicities $j\left(X, Y ; C_{i}\right)$ are given by

$$
\begin{aligned}
& C_{1}: x_{0}=x_{2}=0 ; C_{2}: x_{0}=x_{1}=x_{4}=0 ; C_{3}: x_{0}=x_{1}=x_{2}=0 ; \\
& C_{4}: x_{0}=x_{2}=x_{3}=0 ; C_{5}: x_{0}=x_{1}=x_{2}=x_{4}=0 ; \\
& C_{6}: x_{1}=x_{2}=x_{3}=x_{4}=0 ; \text { and } j\left(X, Y ; C_{1}\right)=j\left(X, Y ; C_{3}\right)=j\left(X, Y ; C_{4}\right)=2 \\
& \quad \text { and } j\left(X, Y ; C_{2}\right)=j\left(X, Y ; C_{5}\right)=j\left(X, Y ; C_{6}\right)=1 .
\end{aligned}
$$

This example shows that the imbedded point $C: x_{0}=x_{1}=x_{2}=x_{3}=0$ belonging to $I(X)+I(Y)$ does not appear in our decomposition of $\operatorname{deg}(X)$. $\operatorname{deg}(Y)$ although $\operatorname{dim} C \geq \operatorname{dim} X+\operatorname{dim} Y-n=0$ and there is a "geometrically imbedded" point, namely $C_{5}$. 
Example 7 shows that the following case is possible: The decomposition of $\operatorname{deg}(X) \cdot \operatorname{deg}(Y)$ is stated by using only the isolated components of $X \cap Y$ although $I(X)+I(Y)$ has imbedded components with dimension $\geq \operatorname{dim} X+\operatorname{dim} Y-n$.

Example 7. In $P_{K}^{3}$ we consider subschemes $X$ and $Y$ defined by the following ideals:

$$
I(X)=\left(x_{0}, x_{1}\right) \cap\left(x_{2}, x_{3}\right) \text { and } I(Y)=\left(x_{0}+x_{2}, x_{0}^{2}\right) .
$$

Then $X \cap Y$ has two isolated points, say $C_{1}: x_{0}=x_{1}=x_{2}=0$ and $C_{2}: x_{0}=$ $x_{2}=x_{3}=0$. The theorem does provide that $j\left(X, Y ; C_{i}\right) \geq 2$ for $i=1,2$. Since $4=\operatorname{deg}(X) \cdot \operatorname{deg}(Y) \geq j\left(X, Y ; C_{1}\right) \cdot \operatorname{deg}\left(C_{1}\right)+j\left(X, Y ; C_{2}\right) \cdot \operatorname{deg}\left(C_{2}\right)$ by applying the statement of [17], we see that $j\left(X, Y ; C_{1}\right)=j\left(X, Y ; C_{2}\right)=2$; that is,

$$
\operatorname{deg}(X) \cdot \operatorname{deg}(Y)=j\left(X, Y ; C_{1}\right) \cdot \operatorname{deg}\left(C_{1}\right)+j\left(X, Y ; C_{2}\right) \cdot \operatorname{deg}\left(C_{2}\right) \cdot
$$

We note that $I(X)+I(Y)$ has an imbedded component of dimension $\geq \operatorname{dim} X$ $+\operatorname{dim} Y-n=-1$. We want to conclude with an example mentioned in our introduction.

Example 8. In $\boldsymbol{P}_{K}^{2}$ we consider subschemes $X, Y$ defined by $I(X)=$ $\left(x_{1}^{2} x_{2}\right)$ and $I(Y)=\left(x_{1} x_{2}^{2}\right)$. The isolated components of $X \cap Y$ are the lines $C_{1}: x_{1}=0$ and $C_{2}: x_{2}=0$. Since the local rings $A\left(X ; C_{1}\right)$ and $A\left(Y ; C_{2}\right)$ are not regular, our criterion for intersection multiplicity one yields $j\left(X, Y ; C_{i}\right)>1$ for $i=1,2$. However, in the intersection theory of Fulton and MacPherson using the diagram

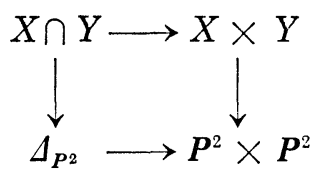

each line contributes 1 to the Bezout number $\operatorname{deg}(X) \cdot \operatorname{deg}(Y)=9$ (see [3], Example 11.3.3, (d)).

\section{REFERENCES}

[0] Achilles, R., On the intersection multiplicity of improper components in algebraic geometry, Beiträge Algebra Geom., 19 (1985), 113-129.

[1] Avramov, L. L., Homology of local flat extensions and complete intersection defects, Math. Ann., 228 (1977), 27-37.

[2] Budach, L. und W. Vogel, Cohen-Macaulay Moduln und der Bezoutsche Satz, Monatsh. Math., 73 (1969), 97-111.

[ 3 ] Fulton, W., Intersection theory. Ergebnisse der Mathematik, 3. Folge. SpringerVerlag New York-Heidelberg-Berlin-Tokyo, 1984. 
[4] Gröbner, W., Moderne algebraische Geometrie, Springer-Verlag Wien und Innsbruck, 1949.

[ 5 ] Grothendieck, A., Éléments de géométrie algébrique, I.H.E.S., Publ. Math., Paris, No. 24 (1965).

[6] Herzog, J. und E. Kunz, Der kanonische Modul eines Cohen-Macaulay-Rings, Lecture Notes in Math., No. 238, Springer-Verlag Berlin-Heidelberg-New York, 1971.

[7] Kiehl, R. und E. Kunz, Vollständige Durchschnitte und p-Basen, Arch. Math. (Basel), 16 (1965), 348-362.

[ 8 ] Lazarsfeld, R., Excess intersection of divisors, Compositio Math., 43 (1981), 281296.

[ 9 ] Matsumura, H., Commutative algebra, Benjamin, New York, 1970.

[10] Nagata, M., Local rings, Interscience Publ., New York, 1962.

[11] Patil, D. P. and W. Vogel, Remarks on the algebraic approach to intersection theory, Monatsh. Math., 96 (1983), 233-250.

[12] Samuel, P., Méthodes d'algèbre abstraite en géométrie algébrique, Ergebnisse der Mathematik, Neue Folge. Springer-Verlag Berlin-Göttingen-Heidelberg, 1955.

[13] Selder, E., Lokale analytische Schnittmultiplizitäten, Dissertation, Universität Osnabrück, 1981.

[14] Serre, J.-P., Algèbre locale. Multiplicités. Lecture Notes in Math., No. 11, SpringerVerlag Berlin-Heidelberg-New York, 1965.

[15] Severi, F., Osservazioni sul Restsatz per le curve iperspaziali, Atti della R. Acc. delle Scienze di Torino, 1908.

[16] _- Il concetto generale di multiplicita della soluzioni per sistemi de equazioni algebriche e la tecria dell'eliminazione, Annali di Math., 26 (1947), 221-270.

[17] Stückrad, J. and W. Vogel, An algebraic approach to the intersection theory. In: The curves seminar at Queen's Vol. II, 1-32. Queen's papers in pure and applied mathematics, No. 61, Kingston, Ontario, Canada, 1982.

[18] Vogel, W., Lectures on results on Bezout's Theorem, Lecture Notes, Tata Institute of Fundamental Research of Bombay (Notes by D. P. Patil), Springer-Verlag Berlin-Heidelberg-New York, Tokyo, 1984.

[19] Waerden, B. L. van der, Algebra, 5. Aufl. der Modernen Algebra, 2. Teil. SpringerVerlag Berlin-Heidelberg-New York, 1967.

[20] Weil, A., Foundations of algebraic geometry, Amer. Math. Soc., Providence, R. I., 1962.

[21] Zariski, O. and P. Samuel, Commutative algebra, Vol. I and II. D. van Nostrand Company, Princeton, 1958 and 1960.

Craig Huneke

Purdue University

Department of Mathematics

West Lafayette, Indiana 47907

USA

Rüdiger Achilles

Wolfgang Vogel

Martin-Luther-University

Department of Mathematics

4010 Halle

German Democratic Republic 